\title{
Nuevos enfoques para viejos problemas. Construcción de alternativas para la gestión ambiental de la Cuenca de Laguna del Sauce
}

\section{NEW APPROACHES TO OLD PROBLEMS. CONSTRUCTION OF ALTERNATIVES FOR THE ENVIRONMENTAL MANAGEMENT OF THE LAGUNA DEL SAUCE BASIN}

\author{
Cabot Ma. E.* Cappuccio, L. * Guillén, J. * Pastorino, G. * Pirez, M. * Silvera, N. *1 \\ babu.cabot@gmail.com \\ Recibido: 05/06/2020 Aceptado: 21/06/2020
}

\begin{abstract}
Resumen
En los últimos años, se han generado una serie de arreglos institucionales novedosos para el país, como la creación de Comisiones de Cuenca y la aprobación de Planes de Acción para la gestión de cuencas que son proveedoras de agua potable y han presentado problemáticas vinculadas a la calidad del recurso. Dentro del conjunto de medidas que integran los Planes de Acción, se incluyen regulaciones ambientales a la actividad agropecuaria, con la finalidad de priorizar en calidad y cantidad el servicio de provisión de agua potable. En este trabajo abordamos el análisis del Plan de Acción elaborado para la Cuenca de Laguna del Sauce, con el objetivo de identificar avances, barreras y alternativas, con respecto a las medidas que buscan controlar los aportes difusos de la actividad agropecuaria. Se realizaron entrevistas a integrantes de la Comisión de Cuenca de Laguna del Sauce (CCLS), Mesa de Desarrollo Rural (MDR), productores y gremiales rurales. Complementando la información por medio de observación participante en talleres, reuniones y revisión bibliográfica. A partir de la evidencia generada, por un lado, se discutieron los problemas identificados como limitantes para la aplicación de las medidas, el rol de los distintos actores y la posición del sector agropecuario en este contexto. Por otro lado, se analizó la correspondencia entre los avances en la gestión y la transición entre paradigmas de gestión.
\end{abstract}

Palabras clave: Gestión de cuencas; Paradigma de comando y control; Manejo adaptativo; Contaminación de origen difuso

\begin{abstract}
Recently, a number of innovative institutional arrangements have been approved in Uruguay, such as the creation of the "Basin Commissions" and "Action Plans" in order to manage watersheds involved in potable water supply, which have had quality problems in the past and present. The measures taken within the Action Plans include environmental regulations for agricultural practices, among others, in order to ensure a good quality and quantity for the supply of drinking water. In this respect, our work addresses the analysis of the Action Plan developed for the "Laguna del Sauce Basin", in order to identify progress, barriers and alternatives generated as a result of the measures taken to seek control diffuse outputs from agricultural activities into the watershed. Interviews were conducted with members of the "Laguna del Sauce Basin Commission", a Rural Development Committee (an organization that serves as a linkage between governmental authorities and rural organizations), local rural producers and rural guild organizations. This information was complemented with that generated by participant observation methodology in workshops, meetings and bibliographic review on the subject. Therefore, the discussion is set upon the main problems that limit the application of the measures, the role that the different territorial entities and locals have on the matter and the position that participants from the agricultural sector have in this context. On the other hand, the advances in management in correspondence to the transition of the management paradigm were discussed
\end{abstract}

\footnotetext{
1 *Centro Universitario Regional Este-Universidad de la República

Tekoporá ${ }^{\circledR}$. Centro Universitario de la Región Este. Universidad de la República (C) Cabot et al. (2020)

Este es un artículo de Acceso Abierto distribuido bajo licencia Creative Commons (CC BY NC 4.0)
} 


\section{Introducción}

Dentro de los problemas ambientales más importantes para los cuerpos de agua dulce, los procesos de eutrofización son considerados un problema central, pudiendo afectar su uso y sus servicios ecosistémicos (Hutchinson, 1973; Wetzel, 2001; Smith, 2003; Carpenter, Stanley y Vander Zanden, 2011). La eutrofización implica el aumento en la concentración de nutrientes, principalmente nitrógeno y fósforo en los sistemas acuáticos, pudiendo generar varios problemas en la calidad de agua, entre ellos, la aparición de floraciones de cianobacterias (Likens, Bormann, Johnson, Fisher y Pierce, 1970; Wetzel, 2001). Estos nutrientes que llegan a los cuerpos de agua pueden provenir tanto de fuentes puntuales como de fuentes difusas (Carpenter et al., 1998; Wetzel, 2001). Las fuentes puntuales se originan en sitios concretos, lo que implica cierta frecuencia o temporalidad, siendo un ejemplo de fuente puntual la descarga de un efluente urbano, industrial o agrícola (Carpenter et al., 1998; Ritter y Shirmohammadi, 2000). Por otro lado, las fuentes no puntuales o difusas pueden provenir de más de un sitio dentro de la cuenca y su llegada al cuerpo de agua puede darse por distintas vías como la precipitación, deposición atmosférica, drenaje, escorrentía superficial o filtración (Ritter y Shirmohammadi, 2000; Wetzel, 2001). Dentro de las fuentes difusas se pueden incluir los desechos generados por las actividades agropecuarias por el uso de insumos tales como los fertilizantes, aditivos químicos y otros de la actividad pecuaria extensiva (Carpenter et al., 1998; Ritter y Shirmohammadi, 2000; Smith, 2003). En Uruguay el fenómeno de la eutrofización ha sido registrado en diversos cuerpos de agua, siendo un problema central porque afecta la calidad de agua para diversos usos (Conde y Sommaruga, 1999; Scasso y Mazzeo, 2000; Mazzeo et al., 2002).

En el Departamento de Maldonado, se han registrado fenómenos de floraciones de cianobacterias en la Laguna del Sauce que se han intensificado y acelerado en los últimos tiempos (Steffen e Inda, 2010). Siendo esta la principal fuente de agua para potabilizar, este fenómeno ha generado diversas interferencias en la provisión del servicio de agua potable para el departamento (Unidad Reguladora de Servicios de Energía y Agua, 2017). Una de ellas, en marzo del 2015 generó un evento de olor y sabor en el agua que se determinó como pérdida de calidad e imposibilitó el consumo de agua por parte de la población (Unidad Reguladora de Servicios de Energía y Agua, 2017). Dicho evento derivó en alarma pública y generó importantes movimientos sociales y políticos a distintas escalas, resultando en la eximición del pago de los servicios de Obras Sanitarias del Estado (OSE) a todo el departamento (Galán, 2015).

Posteriormente se aprobó por resolución del Ministerio de Vivienda, Ordenamiento Territorial y Medio Ambiente (MVOTMA) un Plan de Acción para la Cuenca de la Laguna del Sauce basado en el trabajo de la Comisión de Cuenca Laguna del Sauce (CCLS) y en algunas las 
medidas adoptadas para la Cuenca del Santa Lucía en un plan de acción propuesto en el 2013. Este plan contiene doce medidas que apuntan a la preservación del cuerpo de agua, a controlar las fuentes que aportan contaminación a la Laguna y a mantener la disponibilidad del recurso como fuente de agua potable (Resolución MVOTMA 617a/2015).

En este trabajo se aborda el análisis del Plan de Acción elaborado para la Cuenca de Laguna del Sauce, con el objetivo de identificar avances, dificultades y nuevas alternativas con respecto a las medidas y estrategias que buscan controlar los aportes difusos de la actividad agropecuaria, incorporando las perspectivas del manejo adaptativo y el co-manejo. Para esto se plantearon los siguientes objetivos específicos: (1) Analizar los ámbitos vinculados a la gestión del territorio, identificando los actores y sus relaciones en los procesos de toma de decisión claves; (2) Evaluar las medidas del Plan de Acción adoptado en el año 2015 por el nivel nacional y las medidas precautorias del nivel departamental directa o indirectamente relacionadas con la contaminación de origen difuso; (3) Identificar avances, barreras y oportunidades en el control del aporte difuso; (4) Elaborar propuestas alternativas para incentivar la implementación y el ajuste de las medidas de control de contaminación de origen difuso para los casos en los que se detecten dificultades.

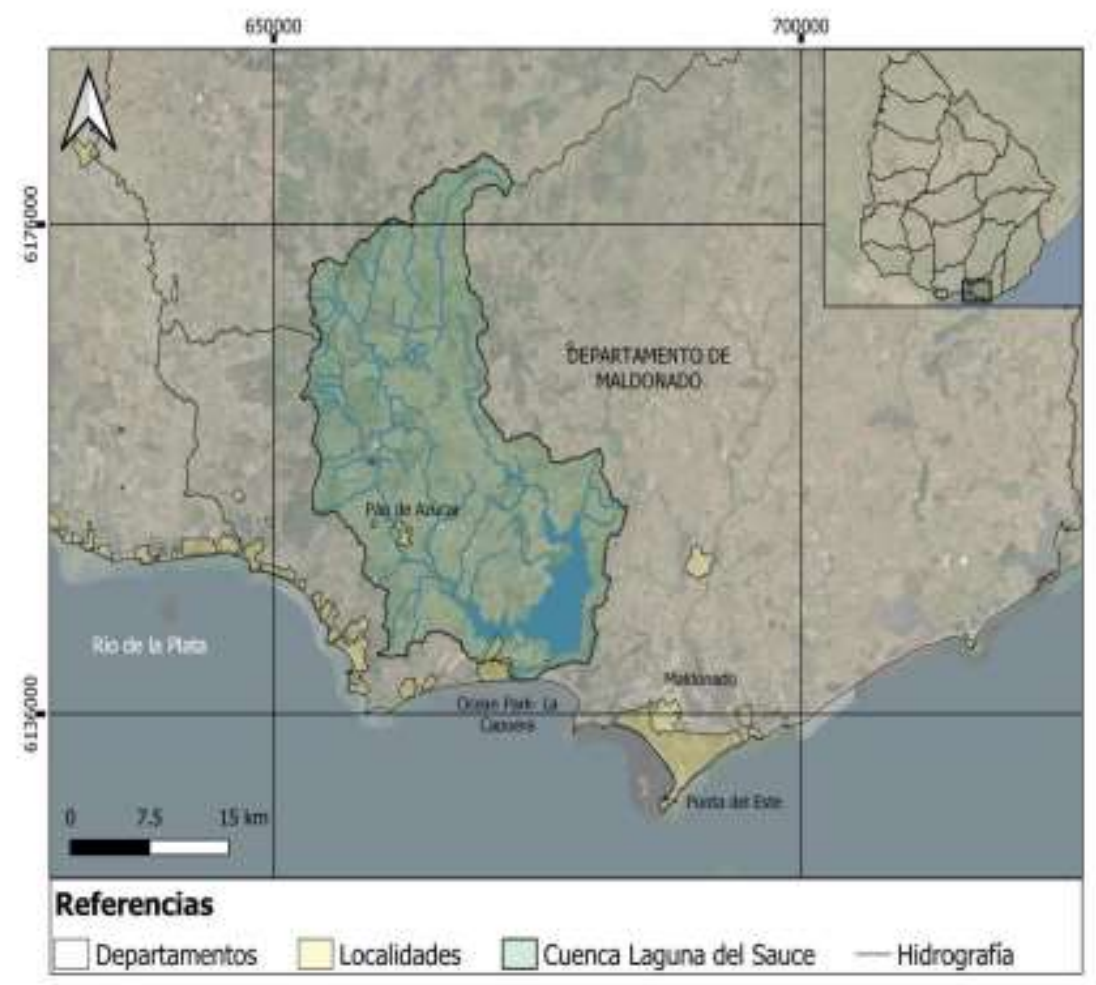

Figura 1. Localización de la Cuenca de Laguna del Sauce. Fuente: elaboración propia en base a IDE 2019 y ESRI 2019.

\section{Área de estudio}

La Cuenca de Laguna del Sauce abarca $709 \mathrm{~km}^{2}$, correspondiendo al $15 \%$ del territorio del Departamento de Maldonado, ubicándose al centro sur occidental del mismo.

El espejo de agua de la Laguna del Sauce es parte de un sistema de tres lagunas conectadas: Del Sauce (4.045 ha), De Los Cisnes (205 ha) y Del Potrero (411 ha). Sus dos afluentes principales son el arroyo Pan de Azúcar y el arroyo del Sauce, siendo el arroyo del Potrero su desagüe natural hacia el Río de la Plata. En la conexión de la Laguna con el arroyo El Potrero se encuentra una represa desde mediados del siglo XX que ha aumentado el nivel de agua en la laguna e interrumpe la dinámica de entrada de agua salina (Rodríguez, Méndez, Inda, Lagomarsino y Steffen, 2010). 
La Laguna del Sauce ha sido definida como un lago eutrófico, con elevados niveles de fósforo en varias ocasiones (Steffen e Inda, 2010). Dentro del sistema de tres lagunas, la del Potrero presenta un crecimiento elevado de la vegetación acuática (macrófitas), en tanto las otras lagunas (Del Sauce y De los Cisnes) presentan floraciones ocasionales de cianobacterias, principalmente durante el verano, con potencial de presencia de cianotoxinas (Crisci et al., 2017). El volumen de cianobacterias presente en Laguna del Sauce se ha encontrado eventualmente por encima del nivel de alerta fijado por la OSE, a su vez las floraciones se han dado próximas a la toma de potabilización, por lo que representan un importante riesgo para el tratamiento de potabilización (González-Madina et al., 2017).

La Laguna del Sauce es la principal fuente de agua potable del este del país (Crisci et al., 2017). Provee de agua potable a más del $95 \%$ de la población fija del Departamento de Maldonado (140.000 personas) y flotante (puede superar las 600.000 personas en temporada estival) del departamento (Maldonado, Punta del Este, San Carlos, Piriápolis y sus zonas periféricas) desde 1970 (Steffen e Inda, 2010; Instituto Nacional de Estadística, 2011).

En cuanto a la cobertura del suelo de la cuenca, según Taveira et al. (2018) el campo natural representa el 53,9\%, seguido por la forestación con 14,8\% de la superficie, a partir de un análisis de la carta de uso de suelo extraída de SIT-MVOTMA, con una clasificación según Land Cover Classification System (LCCS), 2015. La principal actividad productiva en la cuenca es la ganadería de carne, la mayoría de los predios desarrollan la etapa de cría de ganado, con una producción sobre campo natural y pasturas regeneradas (Taveira et al., 2018).

\section{Metodología}

Como estrategia de investigación se optó por combinar diversos métodos que incluyeron el análisis a partir de información primaria y secundaria en torno al Plan de Acción y al funcionamiento de los ámbitos de gestión del territorio. Para abordar los objetivos se realizaron entrevistas a integrantes de la Comisión de Cuenca de Laguna del Sauce (CCLS), de la Mesa de Desarrollo Rural (MDR), productores y gremiales rurales. La información recabada de las entrevistas se complementó por medio de observación participante en talleres, reuniones y revisión bibliográfica. Las distintas metodologías empleadas apuntaron a evaluar las medidas a partir de las perspectivas de los actores y su vínculo con la cuenca y la problemática; poniendo el foco especialmente en conocer el grado de implementación de las medidas, su comunicación a las partes vinculadas (ya sea por tener responsabilidades en la aplicación o fiscalización) y la valoración de las medidas en sí, considerando su posibilidad de ejecución real y buscando conocer las posibilidades de los productores para ejecutar las medidas.

Para ellos, se realizaron 19 entrevistas, de las cuales 11 fueron realizadas a instituciones estatales, 7 a productores rurales y representantes de las sociedades de fomento 
rural y 1 a una organización vecinal.

En el transcurso del proyecto se participó de nueve instancias de talleres vinculados a la gestión y planificación de la cuenca de Laguna del Sauce. Además, se participó en una de las reuniones de la Mesa de Desarrollo Rural de Maldonado y a tres sesiones de la CCLS.

\section{Resultados}

Se detectó un desajuste entre la normativa y su aplicación en el territorio, producido por su creación desde instituciones formales a nivel nacional. El carácter centralizado en el desarrollo de la normativa implicó que a nivel de territorio, aquellos actores responsables de cumplir las medidas, en su mayoría desconocían dicha resolución y no se consideraban partícipes en la decisión. Lo que se consideró como un desafío a la hora de generar involucramiento de los actores que tienen que implementar las medidas.

Las entrevistas y asistencias a talleres permitieron detectar una ausencia de control territorial, que se vió acrecentada por la dificultad existente para consolidar un equipo que trabaje en el territorio de forma sostenida en el tiempo. Además, se constató que existía una falta de comunicación importante entre todos los niveles de actuación. A pesar de esto, diversas instituciones siguieron apostando a paradigmas donde primaba el control y la fiscalización como elementos dominantes. Sin embargo, también se detectó que las medidas lograron un aval importante en las instituciones estatales y en los último tiempo de la investigación el Ministerio de Ganadería, Agricultura y Pesca (MGAP) pasó a asumir un rol protagónico, siendo una entidad que propone estrategias para avanzar en la implementación del Plan de Acción, aprovechando su vínculo con los productores y generando incentivos económicos a través del Banco Mundial y medidas de apoyo técnico para efectivizar la aplicación de las medidas.

Se caracterizaron dos ámbitos de gestión implicados en la aplicación del plan de acción: la Comisión de Cuenca de Laguna del Sauce y La Mesa de Desarrollo Rural. En relación al primero se identificó que la representación es principalmente del sector institucional, evidenciándose una baja representación de la sociedad civil y organizaciones vinculadas a las actividades económicas del departamento. Por otro lado, aquellos actores sociales que han participado en los ámbitos mencionados, aducen problemas para la implementación o la aplicación de lo allí planteado, considerando que las medidas discutidas deben materializarse.

En cuanto a la valoración general de los espacios, se mantiene una visión positiva de la CCLS por parte actores institucionales y los representantes de la sociedad civil, aunque esta última menciona insatisfacción por los tiempos de trabajo en relación a los logros y en algunos casos que los planteos o medidas acordadas en el espacio no han podido ser aplicadas. La Comisión de Cuenca tiene un carácter no vinculante, esto limita su capacidad de acción y esta 
capacidad limitada se visualiza de forma clara por las instituciones que participan de ella. Sin embargo, esto genera disconformidad para la sociedad civil, alegando la falta de resultados.

En general los actores visualizan a la CCLS como el ámbito responsable de dar respuesta a los problemas detectados y de obtener resultados visibles respecto a la problemática de calidad de agua. Por lo que este espacio se visualiza a nivel institucional como un espacio de coordinación entre los niveles nacional y departamental.

Por otro lado, en la MDR se encontraron visiones positivas reincidentes y una valoración del espacio como provechoso en términos de productividad. Se constató que el MGAP, la Dirección de Desarrollo Rural de la Intendencia, varias asociaciones de productores y productores independientes participaron de este espacio. La valoración de la MDR fue catalogada como un espacio central en la vinculación entre actores institucionales y sociales del medio rural, destacándose la importancia de este ámbito confortable para la expresión de los actores involucrados.

Aunque se identificó al principio de la presente investigación una fuerte presencia de representantes del MGAP en territorio a través de esta mesa, no se encontraron vínculos o formas de interacción directas entre la misma y la CCLS. Asimismo, aunque se detectó un solapamiento territorial entre ambos ámbitos de gestión, siendo el alcance departamental de la MDR convergente al de la CCLS, se observó que los orígenes normativos de ambos difieren y por lo tanto no se establece una vinculación directa entre los espacios. Sin embargo, la incorporación de la participación uno de los representantes de la Mesa de Desarrollo Rural en las reuniones de la CCLS en el correr del año 2017 favoreció e impulsó la comunicación y planificación del territorio a nivel local, pudiendo marcar una nueva etapa en la gestión del territorio.

Respecto a la evaluación de las medidas del Plan de Acción, se identificaron grandes dificultades tanto en su implementación como en su ejecución. Se destaca el desconocimiento del Plan de Acción y sus requisitos específicos por parte del sector rural en el momento en que se llevó a cabo el presente estudio. De las siete entrevistas realizadas a productores y a representantes de organizaciones rurales presentes en el territorio, un solo productor de los entrevistados tenía conocimiento de las medidas, de manera extraoficial, ya que no se enteró por medio de las instituciones competentes. Sin embargo, también se registró que la comunicación del plan de acción a los actores en el territorio fue uno de los principales avances en su implementación según algunos actores institucionales. A pesar de que los productores entrevistados mencionaron el desconocimiento del Plan de Acción, al ser aprobado mediante resolución ministerial este tiene carácter jurídico vinculante y empieza a aplicarse a partir de su publicación en el Diario Oficial (Impresiones y Publicaciones Oficiales, 2015). Asimismo, se registró la existencia de un evento de difusión del Plan en sitios institucionales de los organismos estatales (Presidencia, 2015; Ministerio de Vivienda, Ordenamiento Territorial y Medio Ambiente, 2015) y dicha información fue presentada por distintos medios de prensa siguiendo la problemática de la Laguna del Sauce (Muñoz, 2015; 
Camboni, 2015).

El caracter "top-down" con el que fue creado el Plan de Acción tuvo una repercusión negativa en la valoración del mismo por parte de algunos productores entrevistados y actores dentro de la CCLS, puesto que las medidas fueron elaboradas sin la participación del sector productivo rural, siendo estas generadas por el MVOTMA a nivel central a partir de la crisis de calidad de agua - incorporándose propuestas elaboradas años antes por la CCLS durante un período con baja representatividad del sector rural. Esto punto se considera pertinente sobre todo por el largo período de tiempo que se tomó entre la formulación del plan y su comunicación a los principales actores implicados en acatarlo.

Luego de la comunicación del plan por medio de las entrevistas se registró preocupación por parte de los productores y gremiales entrevistados, que manifestaron reiteradamente la posibilidad de encontrar dificultades a la hora de aplicar las medidas del plan, fundamentalmente por las limitantes económicas de predios de la zona y la necesidad de invertir en infraestructura para atender las medidas formuladas, entre otras cuestiones. Aparte de estos cuestionamientos de cómo operar para acatar con las medidas se detectaron diferencias conceptuales entre entrevistados sobre aspectos fundamentales en la aplicación de las medidas, tales como el rol de los aportes difusos en la problemática, las buenas prácticas, la contaminación y los impactos de las actividades humanas sobre la Laguna; conceptos cuya discusión se presenció incluso entre técnicos y a la interna de algunas instituciones y espacios como la CCLS. Se identificó por lo tanto que varios actores declaran en falta hacer definiciones que permitan la comprensión de la problemática, lo que también engloba al debate sobre las responsabilidades institucionales y de los actores presentes en la cuenca. En los talleres observados, también se registraron posiciones de los técnicos y responsables gubernamentales a favor de promover el debate sobre las medidas a implementar, evaluando sus posibilidades reales de ejecución y los apoyos que serían necesarios.

A partir de la observación del espacio y de las valoraciones realizadas por los entrevistados, se visualiza la existencia de la CCLS como una estructura puente entre instituciones formales y centralizadas y otras con mayor despliegue territorial, gobiernos locales y grupos de la sociedad civil organizada. Para algunos actores sigue siendo necesaria una reflexión al respecto de cómo internalizar las discusiones y medidas tratadas en la Comisión, en los organismos de poder formal que tienen capacidad de acción legislativa y práctica. También se refuerza una demanda desde representantes de la sociedad civil, de que su opinión sea considerada, factor que se estima central para mantener en el tiempo la participación de estos sectores dentro de la comisión y con el fin de legitimar las decisiones adoptadas. Los distintos actores entrevistados reconocen a su vez la importancia en la generación de conocimiento académico, pero persiste un reclamo desde los productores de trascender el diagnóstico y anteponen los planteos para abordar soluciones a la problemática y generar cambios en la situación.

Es importante destacar, que este trabajo se desarrolló en un momento particular, en 
donde la falta de coordinación y fragmentación institucional comenzó a dejarse de lado, siendo un claro ejemplo de esto, la coordinación para las salidas de difusión concretadas entre varias instituciones. Además de los múltiples talleres realizados en la cuenca, el año 2017 fue un año particular en el que se observaron varios cambios, entre ellos, un mayor involucramiento de actores institucionales considerados claves que permitió avanzar en la aplicación y financiamiento de las medidas vinculadas al sector agropecuario. El involucramiento del MGAP a partir de la difusión de medidas en la MDR y la generación de ayudas técnicas y financieras a partir de los fondos del llamado del MGAP y el Banco Mundial mediante el "Proyecto Desarrollo y Adaptación al Cambio Climático" (Ministerio de Ganadería, Agricultura y Pesca, 2017), representan un avance diferencial que no se había visualizado hasta entonces y que también implica una superación de la fragmentación sectorial.

\section{Discusión}

La participación de la sociedad es clave y una condición necesaria para lograr la efectiva implementación de políticas de aguas (Achkar, Cayssials, Domínguez y Pesce, 2004) y debe contemplar a los actores más allá de una visión ambiental, incorporando los diferentes usos, actividades e intereses (Parés, 2009). En Uruguay el Estado tiene un rol importante en la gestión (Zurbriggen y Lago, 2014), esto en la cuenca se ve reflejado puesto que el principal usuario del agua es la empresa estatal (OSE) y el control recae también en órganos estatales de nivel nacional o departamental. Actualmente las instituciones se encuentran en una fase que más allá de la interacción en la CCLS y otros espacios, centran sus acciones a sus competencias, lo cual muestra que las instituciones mantienen su lógica estructurada de funcionamiento (Pahl-Wostl, 2015). A su vez las instituciones se encuentran permeadas por el paradigma cognitivo-cultural del comando control (Pahl-Wostl, 2007) y siguen depositando la expectativa de éxito en medidas regulatorias y normativas asociadas a instituciones con competencias rígidas, pero que en la práctica no han logrado establecer un control efectivo, por ejemplo de las actividades que generan aportes difusos. Esto implica que no podemos separar las instituciones formales de los paradigmas dominantes (Pahl-Wostl, 2015), existe una íntima relación que se ve expresa en algunas de las entrevistas, respecto a que cualquier acción requiere un esquema de control previamente establecido, ser medible y contar con resultados esperados. Al profundizar en las acciones que se han implementado y en la actuación de los distintos actores, podemos observar distintos paradigmas de gestión. En la actualidad nos encontramos en un momento de transición, donde se comienza a avanzar desde el Comando Control hacia el Manejo Integrado y el Manejo Adaptativo.

\section{Propuestas que emergen del estudio}

Se propone como alternativa al manejo actual de la CCLS la posibilidad de sesionar en distintas localidades de la cuenca, de manera de acercar al común de la población al ámbito de gestión y a su vez acercar a las instituciones a las realidades presentes en distintos sectores de 
la cuenca. Asimismo, lograr una vinculación entre la MDR y la CCLS que favorezca la implementación y ajuste de las medidas, a través de una participación cruzada de un representante de cada ámbito, con la finalidad de generar puentes que potencien la toma de decisiones y el flujo de información.

Se estima necesario realizar acciones en todos los sentidos y trascender la discusión sobre cuál actividad es más o menos responsable sobre la contaminación de la Laguna del Sauce. Una propuesta para abordar esta situación, es la aplicación de un ordenamiento territorial que reconozca como prioridad la conservación del servicio ecosistémico de provisión de agua potable. Esto implicaría reconocer la estrecha relación que existe entre la ordenación territorial, los usos del suelo y la calidad de agua, teniendo en cuenta también la dificultad que supone revertir la eutrofización. A su vez, es fundamental ir más allá del paradigma de control, incorporando un conjunto de herramientas como incentivos y apoyos técnicos que permitan adecuar las medidas de control de aportes difusos a la realidad territorial. La inclusión de incentivos y apoyos para el cumplimiento de los objetivos del Plan de Acción también surge como una estrategia válida, sobre todo considerando las características del sector agropecuario en la cuenca y la necesidad de cambios en las formas de producción y usos del suelo. Esta estrategia implica un mayor involucramiento por distintos actores e instituciones a nivel del territorio y la generación de espacios de diálogo sobre cuestiones específicas que aporten a soluciones aplicables para cada caso.

Previo a ello se estima necesario la generación de consenso, igualar el conocimiento y los saberes dentro y entre instituciones con respecto a cuáles son las buenas prácticas a implementar, cuál es el rol de los aportes difusos en la problemática. Además, la generación de medidas de gestión debe acompañarse de objetivos claros que estén asociados a indicadores de cumplimiento. Para esto es necesario que exista la capacidad de monitoreo y control, no solo con fines punitivos, sino como forma de evaluar la ejecución y efectividad de las medidas adoptadas, que incluyan la evaluación de las acciones y el compromiso de los organismos públicos.

\section{Conclusiones}

Reconocer las complejidades del medio rural implica que sea necesario activar distintos canales de comunicación para llegar a los productores rurales, donde aparecen como aliados clave las distintas gremiales agropecuarias y el MGAP como actor institucional con fuerte presencia y vínculos en el medio rural. La importancia del sector agropecuario en la cuenca amerita un mayor esfuerzo por trabajar en cercanía con los productores y lograr involucramiento, que a su vez haga posible la efectivización de las medidas a partir de que todos los actores comprendan la problemática y entiendan cómo pueden ser parte de la solución. Esta situación refleja la necesidad de una gestión que proponga nuevos esquemas y formas de vinculación entre las organizaciones y personas, dejando de lado estrategias que ya 
han demostrado su inefectividad para el abordaje del problema socio-ambiental.

Al momento de realización del presente trabajo se verificaron avances con la generación de apoyos (financieros y técnicos) hacia los productores rurales, en búsqueda de implementar efectivamente las medidas y reconociendo el papel de la cuenca en la provisión del servicio ecosistémico de agua potable. A su vez se observó un cambio institucional, donde el MGAP adopta un papel central y genera puentes, primero a la interna de sus direcciónes y luego con otras entidades gubernamentales y de la sociedad civil.

Este trabajo pasa a formar parte del cúmulo de aportes sobre la Cuenca de Laguna del Sauce y es un punto en la historia de la evolución institucional para responder a la problemática socio-ambiental. Por un lado se evidencian progresos en puntos que tenían un importante bloqueo en años anteriores, y por otro, persisten problemas de diversa índole que van desde cuestiones operativas y de coordinación, hasta situaciones más profundas producto de distintas concepciones y paradigmas que predominan sobre todo a nivel de las instituciones estatales. La generación de espacios que nuclean diversos actores requiere una puesta en común y acuerdos en base a los conceptos y objetivos perseguidos, promoviendo la construcción de un marco común de saberes.

A su vez, se debe trascender la discusión sobre cuál actividad es más o menos responsable sobre el aporte de nutrientes a la Laguna, siendo necesario comenzar a realizar acciones en todos los sentidos para impedir que la calidad del agua se siga deteriorando. Una forma de abordar esta situación, es desde un ordenamiento territorial que reconozca la prioridad de conservación del servicio ecosistémico de provisión de agua potable. Lo que implica, reconocer la estrecha relación que existe entre la ordenación territorial, los usos del suelo y la calidad de agua, contemplando la dificultad que supone revertir la eutrofización.

Es fundamental ir más allá de los esquemas de control, incorporando otras herramientas que se enfoquen en la cooperación y construcción colectiva por consenso como los incentivos y apoyos técnicos para adecuar las medidas a la realidad territorial. Incluso sería útil que el componente normativo cuente con una mayor flexibilidad para adaptar las exigencias de la normativa según las problemáticas locales, pudiéndose exigir por ejemplo Planes de Uso y Manejo de Suelos con características distintivas en las cuencas destinadas a la provisión de agua potable. Dicho ajuste permitiría establecer buenas prácticas para las actividades agropecuarias en función de las prioridades locales en cuanto a la preservación de los servicios ecosistémicos, buscando promover actividades que integren esta perspectiva y no la mera exclusión de tipos de usos o actividades.

Se considera importante que en todo futuro proceso, ya sea de creación de normativa o de posibles alternativas para mitigar la contaminación de la Laguna del Sauce, las perspectivas y aportes de los sectores productivos y organizaciones sociales territoriales sean 
integrados desde el comienzo del proceso.

Por último, se considera clave que la generación de medidas de gestión se acompañe de objetivos claros que estén asociados a indicadores de cumplimiento; para ello, es necesario que exista capacidad de monitoreo y de control -no sólo con fines punitivos- sino como forma de evaluar la ejecución y efectividad de las medidas adoptadas, que incluyan la evaluación de las acciones y el compromiso de los organismos públicos en primer lugar.

\section{Bibliografía}

Achkar, M., Cayssials, R., Domínguez, A. y Pesce, F. (2004). Hacia un Uruguay

sustentable: Gestión de Cuencas hidrográficas. Programa Uruguay sustentable.

Montevideo: REDES.

Camboni, G. (2015). El estado coordina medidas para atacar cianobacterias en Laguna del Sauce. El Observador. Recuperado de: https://www.elobservador.com.uy/estado-coordinamedidas-atacar-cianobacterias-laguna-del-sauce-n30279.

Carpenter, S.R., Caraco, N.F., Correll, D.L., Howarth, R.W., Sharpley, A.N. y Smith, V. H. (1998). Nonpoint pollution of surface waters with phosphorus and nitrogen. Ecological applications, 8(3), 559-568.

Carpenter, S. R., Stanley, E. H. y Vander Zanden, M. J., (2011). State of the world's freshwater ecosystems: physical, chemical, and biological changes. Annual Review of Environment and Resources, 36, 75-99.

Conde, C. y Sommaruga, R. (1999). A review of the state of limnology in Uruguay. En: R.G.

Wetzel y P. Gopal (Eds.), Limnology in developing countries (pp. 1-31). Nova Delhi:

International Association for Limnology.

Crisci, C., Goyenola, G., Terra, R., Lagomarsino, J. J., Pacheco, J. P., Díaz, I., González-Madina, L., Levrini, P., Méndez, G., Bidegain, M., Ghattas, B. y Mazzeo, N. (2017). Dinámica ecosistémica y calidad de agua: estrategias de monitoreo para la gestión de servicios asociados a Laguna del Sauce. Maldonado, Uruguay. Innotec, (13), 46-57.

Galán, L. (2015). Sobre los problemas del agua potable en Maldonado. La República.

Recuperado de: https://www.republica.com.uy/problemas-del-agua/

González-Madina, L., Pacheco, J. P., Mazzeo, N., Levrini, P., Clemente, J. M., Lagomarsino, J. J., y 
Fosalba, C. (2017). Factores ambientales controladores del fitoplancton con énfasis en las cianobacterias potencialmente tóxicas en un lago somero utilizado como fuente de agua para potabilización Laguna del Sauce, Maldonado, Uruguay. Innotec, (13). 26-35.

Hutchinson, G. E. (1973). Marginalia: Eutrophication: The scientific background of a contemporary practical problem. American Science, 61(3), 269-279.

Impresiones y Publicaciones Oficiales (2015). Resolución MVOTMA 617a/2015.Disponible en: https://www.impo.com.uy/bases/resoluciones-mvotma/617A-2015.

Instituto Nacional de Estadística (2011). Informe final del Censo. Montevideo,Uruguay.

Recuperado de:http://www.ine.gub.uy/documents/10181/35289/analisispais.pdf/cc0282ef$\underline{20114 \mathrm{ed} 8-\mathrm{a} 3 \mathrm{ff}-32372 \mathrm{~d} 31 \mathrm{e} 690}$

Likens, G.E., Bormann, F.H., Johnson, N.M., Fisher, D.W., Pierce, R.S. (1970). Effects of forest cutting and herbicide treatment on nutrient budgets in the Hubbard Brook watershedecosystem. Ecological monographs, 40(1), 23-47.

Mazzeo, N., Clemente, J., García-Rodríguez, F., Gorga, J., Kruk, C., Larrea, D., Meerhoff, M., Quintans, F.,Rodríguez-Gallego, L. y Scasso, F. (2002). Eutrofización, causas, consecuencias y manejo. En: A. Domínguez y R.G. Prieto (Eds.), Perfil Ambiental del Uruguay (pp. 39-55). Montevideo, Uruguay: Nordan-Comunidad.

Ministerio de Ganadería, Agricultura y Pesca (2017). Proyecto Desarrollo y Adaptación al Cambio Climático (Contrato de Préstamo BM 8099-UY) Recuperado de: http://www.mgap.gub.uy/sites/default/files/multimedia/dacc_af_mgas_y_anexos_-_.pdf Ministerio de Vivienda, Ordenamiento Territorial y Medio Ambiente (2015). Plan de acción para la protección de la calidad ambiental y la disponibilidad como fuente de agua potable de la cuenca hidrológica de la Laguna del Sauce. Recuperado de:

https://mvotma.gub.uy/component/k2/item/10010634-plan-de-accion-para-la proteccion-de-la-calidad-ambiental-y-la-disponibilidad-como-fuente-de-agua-potable-de-lacuenca-hidrologica-de-la-laguna-del-sauce

Muñoz, A. (2015). La clave es el manejo. La Diaria. Recuperado de: https://ladiaria.com.uy/articulo/2015/6/la-clave-es-el-manejo/.

Pahl-Wostl, C. (2007). The implications of complexity for integrated resources management. Environmental Modelling \& Software, 22, 561-569. 
Pahl-Wostl, C. (2015). Water governance in the face of global change: from understanding to transformation. Switzerland: Springer International Publishing.

Parés, M. (2009). Participación y calidad democrática: evaluando las nuevas formas de democracia participativa. Barcelona, España: Ariel.

Presidencia de la República (2015). Plan de Acción Cuenca Laguna del Sauce.

Recuperado de:

https://presidencia.gub.uy/comunicacion/comunicacionnoticias/firma-plan-accioncuenca-laguna-del-sauce-ministra-eneida-de-leon-

Ritter, W. F. y Shirmohammadi, A. (Eds.). (2000). Agricultural nonpoint source pollution: watershed management and hydrology. Florida, U.S.A.: CRC Press.

Rodríguez, A., Méndez, G., Inda, H., Lagomarsino, J. y Steffen, M. (2010). Características y problemática de la Laguna del Sauce. En: M. Steffen y H. Inda (Eds.), Bases técnicas para el manejo integrado de Laguna del Sauce y cuenca asociada (pp. 15-17). Maldonado, Uruguay: Universidad de la República e Instituto Sudamericano para Estudios sobre Resiliencia y Sostenibilidad (SARAS).

Scasso, F. y Mazzeo, N. (2000). Ambientes acuáticos urbanos. En: A. Domínguez A. y R.G. Prieto (Eds.), Perfil Ambiental del Uruguay/2000 (pp. 205-218).Montevideo, Uruguay: NordanComunidad.

Smith, V. H. (2003). Eutrophication of freshwater and coastal marine ecosystems a global problem. Environmental Science and Pollution Research, 10(2), 126-139.

Steffen, M., e Inda, H. (2010). Bases técnicas para el manejo integrado de Laguna del Sauce y cuenca asociada. Montevideo, Uruguay: Universidad de la República y South American Institute for resilience and Sustainability Studies (SARAS).

Taveira, G., Bianchi, P., Díaz, I. e Inda, H. (2018). ¿Cuáles son los principales usos del suelo actuales y tendenciales en la cuenca de Laguna del Sauce?. En: P. Bianchi, G. Taveira, H. Inda y M. Steffen (Eds.), Aportes para la rehabilitación de la Laguna del Sauce y el ordenamiento territorial de su cuenca (pp.47-59). Maldonado, Uruguay: Instituto Sudamericano para Estudios sobre Resiliencia y Sostenibilidad (SARAS).

Unidad Reguladora Servicios Energía y Agua.(2017). Informe de situación de las medidas que se están implementado para el aseguramiento de la potabilización del 
agua del sistema de abastecimiento de Montevideo y Laguna del Sauce. Montevideo. URSEA.

Recuperado de: http://www.ursea.gub.uy/wps/wcm/connect/df7da66d-a66b-4c81-879ad64e6c6f59a6/InformeFinal-v3.2.pdf?MOD=AJPERES\&CVID=lOyRl6q

Wetzel, R.G. (2001). Limnology: lake and river ecosystems. California, United States of America: Elsevier.

Zurbriggen, C., y Lago, M. G. (2014). Innovación y co-creación. Nuevos desafíos para las políticas públicas. Revista de Gestión Pública, 3(2), 329-361. 